\title{
The association between minor recurrent aphthous stomatitis (RAS), children's poor oral condition, and underlying negative psychosocial habits and attitudes towards oral hygiene
}

\author{
S. Tecco ${ }^{1 *}$ D, S. Sciara ${ }^{2}$, G. Pantaleo ${ }^{2}$, A. Nota ${ }^{3}$, A. Visone ${ }^{4}$, S. Germani ${ }^{4}$, E. Polizzi ${ }^{4}$ and E. F. Gherlone ${ }^{1}$
}

\begin{abstract}
Background: Minor Recurrent Aphthous Stomatitis (RAS) represents a disease which is very difficult to prevent. This case-control study focused on possible associations between minor Recurrent Aphthous Stomatitis in children, their oral health, and underlying behavioral indexes of children's attitudes and habits pertaining to (home) oral hygiene, with the further goal of enabling the dentist to prevent these specific kind of lesions, both from a clinical and a broader psychosocial perspective.
\end{abstract}

Methods: Four hundred one school-children (5-10 years old) in Milan (Italy) were submitted to an intra-oral examination, and interviewed with the aid of a brief psychosocial questionnaire.

Results: At the clinical level, statistically significant associations were observed between the presence of decayed teeth and minor Recurrent Aphthous Stomatitis (Odds Ratio: 3.15; 95\% Cl: lower limit 1.06; upper limit: 9.36; Z-test: 2.07, $p=0.039$; Chi-square $=4.71, p=0.030$ ), and between the Decayed Missing or Filled Teeth (DMFT) index and minor aphthous stomatitis (Odds Ratio: 3.30; 95\% Cl: lower limit 1.13; upper limit: 9.67; Z-test $=2.18, p=0.029$; Chi-square = 5.27; $p=0.022$ ), both results pointing to a significant increase - by circa 3 times - in the risk of developing minor Recurrent Aphthous Stomatitis in children exposed to the two above-identified factors (i.e., the presence of decayed teeth and a clearly compromised oral condition, as signaled by the DMFT index), if compared with the risk run by their non-exposed counterparts. At the psychosocial level of analysis, statistically significant associations were observed (1) between children's practice of spontaneously brushing teeth when not at home and a comparatively lower (i.e. better) Decayed Missing or Filled Teeth index (Chi-square: 8.95; $p=0.011$ ), and (2) between receiving parental aid (e.g., proper brushing instructions) while practicing home oral hygiene and a significantly reduced presence of decayed teeth (Chi-square $=5.40 ; p=.067$; Spearman's Rho, $p=.038$ ). Further, significant associations were also observed between children's reported severity of dental pain and both (a) the presence of decayed teeth (Chi-square $=10.80 ; p=0.011$ ), and (b) children's (poor) oral health condition as expressed by the Decayed Missing or Filled Teeth index (Chi-square $=6.29 ; p=0.043$ ). Interestingly, specific lifestyles and social status, showed no systematic association to other clinical or psychological/psychosocial indices. (Continued on next page)

\footnotetext{
* Correspondence: tecco.simona@hsr.it; simtecc@gmail.com

1 Dental School, Vita-Salute San Raffaele University and IRCCS San Raffaele, Via

Olgettina 58, I-20132 Milan, Italy

Full list of author information is available at the end of the article
} 
(Continued from previous page)

Conclusions: These systematic relations suggest that, in the presence of Recurrent Aphthous Stomatitis in pediatric patients, the dentist should carefully monitor children for potential carious lesions, implement protocols of prevention to control Recurrent Aphthous Stomatitis disease in children affected by caries, and also be particularly aware of the right or wrong habits children may acquire in the course of continued social exchange with their caregivers and peers.

Keywords: Stomatitis, Aphthous, Caries, Dental, Psychosocial Attitudes, Habits, and Oral Health, Psychosocial Prevention and Control, Applied Social Psychology

\section{Background}

Minor Recurrent Aphthous Stomatitis (minor RAS) represents a disease which is very difficult to prevent. It is a painful oral mucosal condition characterized by recurrent, multiple, small, round ulcers, surrounded by erythematous haloes, with a necrotic base $[1,2]$.

A minor RAS episode is characterized by a group of 2-5 ulcers with a size of less than $1 \mathrm{~cm}$ in diameter. In a period of about 4-14 days the lesions disappear, and there aren't scars [3].

The scientific literature does not clarify the etiology of minor RAS, but it seems to be related to multifactorial causes [3], such as allergy, trauma [4], genetic predisposition, endocrine disorders, stress and anxiety, and microbiota in the mouth $[5,6]$. Minor RAS episodes are often treated with drugs, topical and systemic, that are considered palliative. This treatment is mainly aimed to decrease inflammation and pain [3].

With respect to the psychosocial side of the issue, there is mounting empirical evidence showing that studies and considerations about oral health status, origins and consequences cannot be limited-any longer-only to clinical issues. Both actual and perceived oral health conditions do in fact heavily affect young patients' quality of life [7], especially in the form of social acceptance by peers [8]. Outcomes of this kind become especially significant when one considers that research has repeatedly shown that parents tend to underestimate the influence of poor oral conditions on their children's emotional and social quality of life [9]. This happens while oral health and dento-facial appearance actually play, instead, a pivotal role in contributing to children's self-esteem and proper social functioning-a finding established long time ago in social psychology and clearly relating to social acceptance, also known as the 'what is beautiful is good' stereotype $[10,11]$. Children's self-confidence and smooth social functioning depend heavily on healthy (facial) appearance $[8,9]$. Monitoring children's behavioral attitudes, habits and practices of (home) oral hygiene becomes, thus, of paramount importance, especially because young patients' attitudes, habits and (good/bad) practices of oral hygiene may all critically affect their actual oral status and, in turn, prompt or hinder the manifestation of precarious oral conditions, such as-for instance-those leading to unwanted episodes of minor RAS.

The importance of scrutinizing the joint influenceand relative outcomes-of both clinical and psychosocial factors in health-related interventions has been recently documented both by research conducted in the area of dentistry and dental hygiene $[12,13]$ as well as by research run in other apparently more distant, yet crucial clinical and psychosocial research areas. [14].

Aim of the study: By focusing on the role of specific clinical and psychosocial variables, considered as potential risk factors (i.e., children's right or wrong behavioral attitudes and habits of home oral hygiene, children's lifestyle, the presence of systemic diseases, and children's general oral hygiene conditions), this study examined possible associations between the above variables and the insurgence of episodes of minor RAS. The ultimate goal of the study was to gather and analyze systematic information to help dentists, hygienists and clinicians in developing useful strategies for preventing the manifestation of such lesions, both from a clinical and a broader psychosocial perspective.

\section{Method}

This observational case-control study analyzed the associations between oral aphthosis, specific behavioral indexes of psychosocial attitudes, habits, and practices of oral hygiene, lifestyle, systemic diseases, and oral health in a sample of 401 School children, aged mostly 610 years, in Milan, Italy. Data were collected during a prevention project of celiac disease, implemented through an agreement between the City Administration of Segrate (Milan, Italy) and the Vita-Salute San Raffaele University (Milan, Italy). The STROBE checklist was used to report the main findings of the study. The protocol was approved by the Ethic Committee of the VitaSalute San Raffaele University (Milan, Italy). Data were recorded in full accordance with ethical principles, including the World Medical Association Declaration of Helsinki. 
Data were collected between 15th November 2014 and 21 st January 2015. The team of operators was composed by 4 dentists, 2 dental hygienists and 8 dental students from San Raffaele Hospital, Vita-Salute San Raffaele University, Milan. The team visited all the children of the School, during several classes. In each class, the operators first gave a short talk to the children about the importance of oral health, nutrition and proper oral hygiene. Then, once obtained parental consent, children were visited by our team of dentists. After this careful intra-oral examination, the children underwent a psychosocial interview [15] and asked a few questions, on a questionnaire-basis, about the behavioral component of their personal attitudes and habits towards oral hygiene and related feelings (e.g. "Do you usually brush your teeth, also when you are not at home?", "When brushing your teeth, does someone help and instruct you?", "Did you ever had teeth ache?", etc.), their lifestyles (e.g. the habit of having an usual snack at school, of practicing sports, possessing and using a personal tablet, etc.), and eventually the presence of systemic diseases (food intolerance, celiac disease).

Case/control ascertainment consisted in the detection of minor RAS, based on clinical observation and a careful conversation with the children about the frequency of episodes (see Additional file 1 for details).

The following variables were collected.

Clinical indices:

- Enamel hypoplasia;

- Enamel Hypoplasia of incisors;

- Enamel Hypoplasia of molars;

- Decayed teeth;

- Filled teeth;

- Decayed, Missing and Filled teeth, leading to the DMFT index;

- Fissures sealings.

Psychosocial and medical indices and predictors:

- The behavioral component of attitudes towards home oral hygiene and related feelings, operationalized in form of interview questions with categorical multiple-choice answering format ("Do you usually brush your teeth, also when you are not at home?" [ $1=$ no, never; $2=$ sometimes; $3=$ always], "When brushing your teeth, does someone help and instruct you?" [1=no; $2=$ sometimes; 3 =yes, regularly], "Did you ever had teeth ache?" $[1=$ no, never; $2=$ yes, just sometimes/a bit; $3=$ yes, I did]);

- Possession of a personal tablet, as an indicator of socio-economic status and, presumably, of family wealth;
- Habit of eating snacks at School, and type of snacks more frequently eaten;

- Presence of food intolerance(s);

- Diagnosis of celiac disease;

- Regular practice of sports activities;

- Topical fluoride treatments at the dentist.

All variables were collected and evaluated with the explicit agreement of all operators, both in the case of clinical observations, and with respect to questionnaire answers (see Additional file 1 for details). The sample included all the children in the School.

Data were handled at patient level (see Additional file 1 for details).

Statistical analyses were performed using the SPSS software. The independence vs. association of categorical and ordinal variables was analyzed using the chi-square test and, in some cases, supplemented by the Spearman Rho non-parametric correlation coefficient. Further, odds ratios (ORs), 95\% confidence intervals, and Z-tests were computed to further illuminate, in terms of possible risk factors, the observed associations. The threshold for statistical significance was set at $p=0.05$ for all statistical tests.

\section{Results}

Among 425 potentially eligible children, all enrolled in the School, 401 children obtained parent consent to receive dental visit, and were thus considered eligible participants for the study. Among the eligible children, 14 cases with minor RAS and 387 controls were ascertained. A percentage of $3.5 \%$ of all eligible children (i.e., 14 children out of 401) represents the prevalence of minor RAS in our sample. Missing data or inconsistent data were not disclosed.

Sample's main demographics (age and gender) are reported in Table 1.

Table 1 Age and gender distribution for the whole sample

\begin{tabular}{llll}
\hline & Gender & & \\
\hline Age & Male $\mathrm{n}(\%)$ & Female $\mathrm{n}(\%)$ & Total $\mathrm{n}(\%)$ \\
5 years old & $1(0.25 \%)$ & 0 & $1(0.25 \%)$ \\
6 years old & $45(11.2 \%)$ & $33(8.2 \%)$ & $78(19.4 \%)$ \\
7 years old & $55(13.7 \%)$ & $33(8.2 \%)$ & $88(21.9 \%)$ \\
8 years old & $37(9.2 \%)$ & $49(12.2 \%)$ & $86(21.4 \%)$ \\
9 years old & $37(9.2 \%)$ & $41(10.2 \%)$ & $78(19.4 \%)$ \\
10 years old & $26(6.4 \%)$ & $40(10 \%)$ & $66(16.4 \%)$ \\
11 years old & 0 & $3(0.7 \%)$ & $3(0.7 \%)$ \\
12 years old & $1(0.25 \%)$ & 0 & $1(0.25 \%)$ \\
Total & $202(50.4 \%)$ & $199(49.6 \%)$ & $401(100 \%)$ \\
\hline
\end{tabular}


Further, with respect to the whole sample and on a purely descriptive level, we observed the following general sample characteristics:

- The majority of children practiced regularly some sports, with $34.2 \%$ of the whole sample (137 children out of 401) practicing more than one sports, and only $15.5 \%$ of the whole sample (62 children out of 401) practicing no sports at all;

- More than a third of all interviewed children (39.2\% of the whole sample, 157 children out of 401) possessed a personal tablet for private use, this indicating a comparatively higher social status in every child out of three;

- Five children out of 401 (1.2\% of the whole sample) resulted affected by celiac disease;

- Twenty-five children out of 401 (6.2\% of the whole sample) presented food intolerances, mainly due to milk and dairy products (11 children out of 25).

Further, always with respect to the whole sample, and on a purely descriptive level, we observed also the following characteristics pertaining to children's oral health:

- Prevention appeared to be not very diffused, as 56 children out of 401 ( $14 \%$ of the whole sample) showed sealants on permanent first molars, and only 45 children out of 401 (11.2\% of the whole sample) were used to receive professional topical fluoride treatments in the dental office;

- Eighty children out of 401 (20\% of the whole sample) had decayed teeth;

- Eighteen children out of 401 (4.5\% of the whole sample) had filled teeth;

- Enamel hypoplasia was present in 72 children out of 401 ( $18 \%$ of the whole sample), mostly at the incisive teeth (51 children out of 72 with enamel hypoplasia at the incisive area);

- In our sample of 401 children aged 5-10 years old, prevalence of RAS was $3.5 \%$.

Numbers of participants assigned to each exposure category are reported in Table 2, along with chisquare tests of independence for comparing (sub-)categories of study participants (with Yates' continuity correction, if intra-cell $n \mathrm{~s}<5$ ).

A significant association was observed between the presence of decayed teeth and minor RAS (Odds Ratio $=3.15,95 \% C I=$ lower limit 1.06, upper limit 9.36; Ztest $=2.07, p=0.039$. A chi-square analysis confirmed the observed result, Chi-square $=4.71, p=0.030$ ). In sum, the risk of presenting minor RAS resulted 3.15 times higher for children with decayed teeth than for their non-exposed counterparts.
A significant association was observed also between the DMFT index $>0$ and minor RAS (Odds Ratio $=3.30$, 95\% $C I=$ lower limit 1.13, upper limit 9.67; Z-test $=2.18$, $p=0.029$. The observed result was independently confirmed by a chi-square test, Chi-square $=5.27, p=0.022$ ). In sum, the risk of presenting minor RAS resulted 3.30 times higher for children with DMFT index $>0$ than for their non-exposed counterparts.

At the psychosocial level of analysis-i.e. at the level of analysis explicitly relating psychological and clinical outcomes-statistically significant associations were observed between (a) the habit of brushing teeth when not at home and a comparatively lower (i.e. better) DMFT index (Chi-square $=8.95, p=0.011$ ), and between (b) receiving parental aid (e.g., proper instructions) while practicing home oral hygiene and a comparatively reduced presence of decayed teeth (Chi-square $=5.40, p=.067$; Spearman's Rho, $p=.038$ ).

Further, significant associations were also observed between children's reporting of severity of sensed dental pain and (a) the presence of decayed teeth (Chi-square $=$ 10.80, $p=0.011$ ), and (b) children's oral health condition as expressed by the DMFT index (Chi-square $=6.29$, $p=0.043)$, respectively.

Lifestyles, social status, and systemic diseases showed no association with clinical or psychological indices (all $\left.p_{s}>.05\right)$.

Given the straightforward and plain nature of our research design, and given our declared interest only into primary clinical and psychosocial outcomes, no further subgroup or interaction analyses were performed.

\section{Discussion}

This study aimed to investigate associations between distinct behavioral indexes of psychosocial attitudes, habits, and children's common practices of (home) oral hygiene, general oral health conditions, and the concomitant presence of minor RAS, with the general aim of better understanding, and possibly also preventing, this disorder both from a clinical and a broader psychosocial vantage point.

First, data showed a statistically significant association between decayed teeth and minor RAS.

Only eight children (2.5\%) out of 319 children without decayed teeth were found to have ulcers, while 6 $(7.5 \%)$ out of 80 children with one or more decayed teeth were found to have ulcers $(p=.039)$. The presence of decayed teeth was more frequent in patients with recurrent aphthous compared to healthy subjects. For this reason, the presence of caries seems to predispose more to the development of recurrent aphthous. To be sure, in exposed children the risk of presenting minor RAS resulted to be about 3 times 
Table 2 Raw numbers of exposed vs. non-exposed children ('cases' vs. 'controls') observed in each study category with respect to each of the potential risk factors. P-values refer to statistical procedures testing for the association between the examined factors and Minor Recurrent Aphthous Stomatitis (RAS). \% values are shown in parenthesis

\begin{tabular}{|c|c|c|c|c|}
\hline Factors of Exposure & Categories & $\begin{array}{l}\text { Number (\%) of children among } \\
\text { the } 387 \text { Controls }\end{array}$ & $\begin{array}{l}\text { Number (\%) of children among } \\
\text { the } 14 \text { Cases }\end{array}$ & $\begin{array}{l}\text { Statistical Significance } \\
\text { of Results }\end{array}$ \\
\hline \multirow[t]{3}{*}{ Sports Practice } & No sports at all & $58(15 \%)$ & $4(28.6 \%)$ & \multirow[t]{3}{*}{$p=0.32$, n.s. } \\
\hline & $\begin{array}{l}\text { One single sports } \\
\text { activity }\end{array}$ & $195(50.4 \%)$ & $7(50 \%)$ & \\
\hline & $\begin{array}{l}\text { More than } 1 \text { sports } \\
\text { activity }\end{array}$ & $134(34.6 \%)$ & $3(21.4 \%)$ & \\
\hline \multirow[t]{2}{*}{ Decayed teeth } & No & $311(80.8 \%)$ & $8(57.1 \%)$ & \multirow[t]{2}{*}{$p=0.03^{* * *}$} \\
\hline & Yes & 74 (19.2\%) & $6(42.9 \%)$ & \\
\hline \multicolumn{5}{|l|}{ Usual snacks at School: } \\
\hline \multirow[t]{2}{*}{ a) Milky and dairy } & No & $172(44.4 \%)$ & $7(50 \%)$ & \multirow[t]{2}{*}{$p=0.68$, n.s. } \\
\hline & Yes & $215(55.6 \%)$ & $7(50 \%)$ & \\
\hline \multirow[t]{2}{*}{ b) Sugars and carbohydrates } & No & $84(21.7 \%)$ & $1(7.1 \%)$ & \multirow[t]{2}{*}{$p=0.32$, n.s. } \\
\hline & Yes & $300(77.5 \%)$ & $13(92.9 \%)$ & \\
\hline \multirow[t]{2}{*}{ c) Fruits } & No & 348 (89.9\%) & $13(92.9 \%)$ & \multirow[t]{2}{*}{$p=0.72$, n.s. } \\
\hline & Yes & $39(10.1 \%)$ & $1(7.1 \%)$ & \\
\hline \multirow[t]{2}{*}{ d) Fruit Juices } & No & $359(92.8 \%)$ & $14(100 \%)$ & \multirow[t]{2}{*}{$p=0.30$, n.s. } \\
\hline & Yes & $28(7.2 \%)$ & 0 & \\
\hline \multirow[t]{2}{*}{ e) No snacks } & No & $382(98.7 \%)$ & 14 (100\%) & \multirow[t]{2}{*}{$p=0.67$, n.s. } \\
\hline & Yes & $5(1.3 \%)$ & 0 & \\
\hline \multirow[t]{2}{*}{ Ownership of a personal Tablet } & No & $234(60.6 \%)$ & $9(64.3 \%)$ & \multirow[t]{2}{*}{$p=0.78$, n.s. } \\
\hline & Yes & $152(39.4 \%)$ & $5(35.7 \%)$ & \\
\hline \multirow[t]{2}{*}{ Diagnosis of Celiac disease } & No & $381(98.7 \%)$ & $14(100 \%)$ & \multirow[t]{2}{*}{$p=0.67$, n.s. } \\
\hline & Yes & $5(1.3 \%)$ & 0 & \\
\hline \multirow[t]{2}{*}{ Food Intolerances } & No & $363(93.8 \%)$ & $13(92.9 \%)$ & \multirow[t]{2}{*}{$p=0.89$, n.s. } \\
\hline & Yes & $24(6.2 \%)$ & $1(7.1 \%)$ & \\
\hline \multirow[t]{6}{*}{ Intolerant to what? } & $\begin{array}{l}\text { Milk and dairy } \\
\text { products }\end{array}$ & $10(41.7 \%)$ & $1(100 \%)$ & \multirow[t]{6}{*}{$p=0.93$, n.s. } \\
\hline & Solanaceae & $4(16.7 \%)$ & 0 & \\
\hline & Hazelnuts & $3(12.5 \%)$ & 0 & \\
\hline & Eggs & $3(12.5 \%)$ & 0 & \\
\hline & Fish & $2(8.3 \%)$ & 0 & \\
\hline & Gluten & $2(8.3 \%)$ & 0 & \\
\hline \multirow{2}{*}{$\begin{array}{l}\text { Diagnosis of celiac disease in } \\
\text { family }\end{array}$} & No & 375 (96.9\%) & $12(85.7 \%)$ & \multirow[t]{2}{*}{$p=.13$, n.s. } \\
\hline & Yes & $12(3.1 \%)$ & $2(14.3 \%)$ & \\
\hline \multirow[t]{2}{*}{ Enamel hypoplasia } & No & $316(82.3 \%)$ & $10(71.4 \%)$ & $p=0.30$, n.s. \\
\hline & Yes & $68(17.7 \%)$ & $4(28.6 \%)$ & \\
\hline Enamel hypoplasia of incisors & No & $336(87.3 \%)$ & $12(85.7 \%)$ & $p=0.86$, n.s. \\
\hline & Yes & $49(12.7 \%)$ & $2(14.3 \%)$ & \\
\hline Enamel hypoplasia of molars & No & $364(94.5 \%)$ & $13(92.9 \%)$ & $p=0.79$, n.s. \\
\hline & Yes & $21(5.5 \%)$ & $1(7.1 \%)$ & \\
\hline Filled teeth & No & $368(95.8 \%)$ & $11(84.6 \%)$ & $p=0.22$, n.s. \\
\hline & Yes & $16(4.2 \%)$ & $2(15.4 \%)$ & \\
\hline DMFT index & $\mathrm{DMFT}=0$ & 294 (76.8\%) & 7 (50\%) & $p=0.02^{* * *}$ \\
\hline & $\mathrm{DMFT} \geq 1$ & $89(23.2 \%)$ & $7(50 \%)$ & \\
\hline
\end{tabular}


Table 2 Raw numbers of exposed vs. non-exposed children ('cases' vs. 'controls') observed in each study category with respect to each of the potential risk factors. P-values refer to statistical procedures testing for the association between the examined factors and Minor Recurrent Aphthous Stomatitis (RAS). \% values are shown in parenthesis (Continued)

\begin{tabular}{lllll}
\hline Factors of Exposure & Categories & $\begin{array}{l}\text { Number (\%) of children among } \\
\text { the 387 Controls }\end{array}$ & $\begin{array}{l}\text { Number (\%) of children among } \\
\text { the 14 Cases }\end{array}$ & $\begin{array}{l}\text { Statistical Significance } \\
\text { of Results }\end{array}$ \\
\hline Sealing of teeth & No & $327(84.9 \%)$ & $12(85.7 \%)$ & $p=0.93$, n.s. \\
& $\begin{array}{l}\text { Yes } \\
\text { Sealing of } \\
\text { deciduous teeth }\end{array}$ & $44(14.0 \%)$ & $2(14.3 \%)$ & 0 \\
& No & $342(88.4 \%)$ & $12(85.7 \%)$ & $p=0.90$, n.s. \\
$\begin{array}{l}\text { Topical fluoride treatments in } \\
\text { the dental office }\end{array}$ & Yes & $43(11.1 \%)$ & $2(14.3 \%)$ & 0 \\
& Don't know & $2(0.5 \%)$ & 0 & \\
\hline
\end{tabular}

*** significant at least at $p<.05 ;$ n.s.: not significant. Statistically significant patterns of results are displayed in bold

higher for children with decayed teeth than for their non-exposed counterparts $(O R=3.15, p=0.039)$.

Similarly, data showed also the presence of a statistically significant association between DMFT and minor RAS. Only seven (2.3\%) out of 301 children with $\mathrm{DMFT}=0$ were found to have ulcers, while 7 ( 7 . $3 \%)$ out of 96 of children with DMFT $>0$ were found to have ulcers $(p=.029)$. Ulcers were significantly more frequent in patients with DMFT $>0$ rather than in healthy participants. Again, in exposed children the risk of presenting minor RAS resulted about 3 times higher for children with DMFT $>0$ than for their non-exposed counterparts $(O R=3.30, p=0.029)$.

We also predicted and found, at the psychosocial level of analysis, two further statistically significant associations between (a) the habit of brushing teeth when not at home and a better DMFT index, and between (b) receiving specific parental aid while practicing home oral hygiene and a minor presence of decayed teeth. As negative behavioral attitudes may easily compromise proper practices of oral hygiene and, in turn, lead to decayed teeth and compromised oral status (e.g., minor RAS), the above findings also point to the need of promoting the acquisition of positive attitudes towards oral hygiene by children, through appropriate strategies based on adhoc intervention policies and psychosocial monitoring resources and tools.

By the same token, the significant direct associations between children's reported severity of dental pain with (a) the actual presence of decayed teeth, and with (b) children's general deteriorated oral health condition-as testified to by a comparatively lower DMFT index-, both suggest to pay close attention also to the nuances of children's (un)expressed feelings and sensationssuch as, in our case, pain and teeth ache.

No statistical association between ulcers and filled teeth was detected. This outcome gave us a further confirmation that caries in the active phase (teeth decayed, not the filled teeth), rather than the presence of filled teeth in itself, seem to predispose to the appearance of recurrent aphthous.

Data from this sample did not detect significant associations between RAS and celiac disease, food intolerances, and further indicators of lifestyle such as the customary consumption of snacks at School, the more or less frequent practice of sports, or general socialstatus as inferred by observing whether children possessed a tablet or not.

In the presence of a direct association between RAS and decayed teeth in pediatric patients, the concomitant presence of recurrent aphthae must suggest particular care in evaluating the occurrence of carious lesions, and also the necessity of implementing protocols of prevention to control RAS disease in children affected by caries.

Given the documented association between the psychosocial behavioral indices of a positive general attitude towards oral hygiene and oral health, and given also the documented association between proper oral-health maintenance instructions received by parents and compromised dental status (DFMT), special attention should be paid also to those psychological indices, aspects, and predictors that are able to strengthen children's commitment towards proper hygiene and oral status, on the one hand, and oral health, on the other.

In comparing the results of the present study with those of similar studies and other relevant evidence, we may note that the etiology of RAS ulcers, in the research literature, is still not entirely clear and known. For instance, Sunday et al. [16] conducted research on possible causes of RAS and concluded that the etiology of RAS lesions is still unknown as several local, systemic, immunologic, genetic, allergic, nutritional, and microbial factors have been proposed as causative agents. Also, some medications including immunosuppressive drugs such as calcineurin and mTOR inhibitors have been associated with severe aphthous-like stomatitis. They do not make a direct reference to the presence of cariogenic 
bacteria, but only to the variation of the saliva composition, of saliva, such as $\mathrm{PH}$, which is certainly a factor that correlates with the presence of caries lesions in the active phase in the oral cavity [17-20].

With respect to nutrition and lifestyles, data analysis did not detect significant associations. In the literature, a study by Tarakji et al. [21] assessed the correlation between eating habits and the occurrence of aphthous stomatitis. Their analysis too suggested that, 'Dietary habits have no important role in development of RAS but can be playing a minor role in the pathogenesis of RAS either by causing hypersensitivity or by deficiency of some vitamins and minerals'. As a case in point, their study showed that RAS patients eat acidic $\mathrm{PH}$-containing foods, like oranges and lemons, more frequently than controls, and this habit might have initiated RAS lesions as irritation factors-while other patients might have had some form of hypersensitivity to specific food such as yoghurt and tomato, and spicy food. Also, as research has shown how the choice of different toothpastes critically affects oral health [22], even such a simple habit could possibly concur to the development of RAS and, as such, would be worth of further empirical investigation.

A clear limitation of this study concerns the dental visit that, even if done with all of the necessary attention by our operators, was nevertheless performed in a School, i.e. without the dental chair equipment. In addition, some inaccurate data may have been resulted because of the choice to use a questionnaire that, even if administered with all of the necessary care and attention by our operators, may have rendered it difficult to obtain always sincere and precise answers by children. However, despite this manifest difficulty, the sistematicity of our findings suggests that-at least in the case of children's attitudes and habits-data collection must have had some high degree of reliability.

In this study, we chose to focus on distal behavioral indicators of children's psychosocial habits and attitudes towards oral hygiene, such as their tendency to spontaneously engage in brushing teeth when not at home (i.e., a tendency reflecting children's general positive attitude towards oral care), or their turning to parents in order to receive concrete advice (i.e., again a tendency revealing children's behaviorally-anchored positive attitudes, and signaling their confident and purposive social functioning with respect to proper oral hygiene in a supportive social-relational context). However, from a methodological point of view [23], future research will certainly benefit from extending the scope of empirical studies based almost exclusively on the analysis of behavioral indices such as those spelled out above. Future research should for instance take into account also specific, more proximal, emotional and cognitive facets of young patient's attitudes, prejudices, and habits towards oral hygiene, even implicit [24], because patients' physical and psychological status have clear and direct consequences for perceived quality of life $(Q o L)$ and wellbeing, as consistently shown by recent converging research outcomes stemming both from close [12, 13, 25] and distant $[26,27]$ clinical and psychological research domains. Physical and psychological health are pivotal preconditions for well-balanced and smooth social functioning and social exchange, both at the interpersonal and at the broader societal level [28]. Difficulties children encounter in their everyday oral hygiene should not be considered solely as undesirable obstacles to accurate oral care because, quite on the contrary, moderate difficulties - under proper and specified conditionscan even motivate behavior and related affective responses very strongly, both at the individual and group level [29-33].

Indicators of lifestyle and eating habits can be many, and in this study we have considered only a few. Thus, we cannot exclude that minor RAS might be associated with further lifestyle and/or eating indicators that we did not explicitly consider in this report.

Further, additional clinical and psychosocial studies elucidating the link between RAS/oral hygiene and health-related psychological factors would be extremely relevant and useful in this field. Reserachers could systematically test the idea, for instance, that scanty parental involvement with dental care might be probably causally linked to childrens' poor dental hygiene, and that poor dental hygiene, in turn, could be conducive to both dental decay and RAS, in a two-steps causal chain. If such a causative model were true, then it would easily explain why dental decay and (minor) RAS tend, typically, to be correlated with one another in research. Such a correlation would be explained by the fact that these two variables (i.e., dental decay and RAS) would simply represent clinical manifestations of a common causative factor, within the causal chain hypothesized here. Evidently, however, more research is needed to illuminate the issue.

Finally, our results appear to have a generalizability to the school children population.

\section{Conclusions}

The observed association between minor RAS and teeth decay suggests that, in the presence of recurrent aphthous in pediatric patients, closer attention should be paid to the evaluation of potential carious lesions, by implementing protocols of prevention to control RAS disease in children affected by caries, while considering-at the same time-the manifold attitude-related psychosocial indices, markers, and predictors of children's oral care and, ultimately, oral status and health. 


\section{Additional file}

Additional file 1: Case/control ascertainment, collection of variables, handling of data. Additional information about the methods of the study and the variables considered are reported. (DOCX $15 \mathrm{~kb}$ )

\section{Abbreviations \\ DMFT index: Decayed missing or filled teeth index; RAS: Recurrent aphthous stomatitis}

\section{Acknowledgements}

We acknowledge the Major of Segrate (Milano) who adhered to the Oral Health prevention project proposed by the San Raffaele Hospital. We also thank the Chief Executive of the San Raffaele Hospital (Dr. Nicola Bedin) and the Personnel Director (Dr. Antonio Limardi) for the formalization of the Agreement with the Municipality of Segrate.

\section{Funding}

No funding was received for this study.

\section{Availability of data and materials}

The data that support the findings of this study are available from Vita-Salute San Raffaele University Milan, but restrictions apply to the availability of these data, which were used under license for the current study, and so are not publicly available. Data are however available from the authors upon reasonable request and with permission of Vita-Salute San Raffaele's University partner.

\section{Authors' contributions}

ST: concept, design, clinical procedures, data collection, writing of the article, methodology, critical revisions, approval of the article. SS: concept, design, writing of the article, methodology, statistics, critical revisions, approval of the article. GP: design, research methodology, statistics, writing of the article, critical revisions, approval of the article. AN: writing of the article, critical revisions, approval of the article. AV: clinical procedures, acquisition of data, drafting of the manuscript, accountability for research integrity and accuracy, approval of the article. SG: clinical procedures, acquisition of data, writing and approval of the article. EP: clinical procedures, acquisition and monitoring of data acquisition, drafting of the manuscript, accountability for research integrity and accuracy, final approval of the article. EFG: concept and direction of clinical procedures, critical revision of the manuscript, accountability for research integrity and accuracy, final approval of the article.

\section{Ethics approval and consent to participate}

Written informed consent was signed and obtained for participants and/or their legal representatives (or parents), as appropriate. This study was approved by the Institutional Review Boards of Vita-Salute San Raffaele University

\section{Consent for publication}

Not applicable.

\section{Competing interests}

The authors declare that they have no competing interests.

\section{Publisher's Note}

Springer Nature remains neutral with regard to jurisdictional claims in published maps and institutional affiliations.

\section{Author details}

${ }^{1}$ Dental School, Vita-Salute San Raffaele University and IRCCS San Raffaele, Via Olgettina 58, I-20132 Milan, Italy. ${ }^{2}$ UniSR-Social.Lab [Research Methods], Faculty of Psychology, Vita-Salute San Raffaele University, Milan, Italy. ${ }^{3}$ University of Tor Vergata, Rome, Italy. ${ }^{4}$ Center for Oral Hygiene and Prevention, Dental School, Vita-Salute San Raffaele University and IRCCS San Raffaele, Milan, Italy.
Received: 15 December 2016 Accepted: 16 March 2018

Published online: 13 April 2018

\section{References}

1. Jurge S, Kuffer R, Scully C, Porter SR. Recurrent aphthous stomatitis. Oral Dis. 2006:12:1-21.

2. Burruano F, Tortorici S. Major aphthous stomatitis (Sutton's disease): etiopathogenesis, histological and clinical aspects. Minerva Stomatol. 2000; 49:41-50.

3. Belenguer- Guallar I, Jiménez-Soriano Y, Ariadna Claramunt-Lozano A. Treatment of recurrent aphtous stomatitis. A literature review. J Clin Exp Dent. 2014;6(2):e168-74

4. Wray D, Graykowski EA, Notkins AL. Role of mucosal injury in initiating recurrent aphthous stomatitis. Br Med J (Clin Res Ed). 1981;283:1569-70.

5. Liang MW, Neoh CY. Oral aphthosis: management gaps and recent advances. Ann Acad Med Singap. 2012;41:463-70.

6. Altenburg A, El-Haj N, Micheli C, Puttkammer M, Abdel-Naser MB, Zouboulis CC. The treatment of chronic recurrent oral aphtous ulcers. Dtsch Arztebl Int. 2014;111(40):665-73.

7. Lundgren GP, Karsten A, Dalhoef G. Oral health-related quality of life before and after crown therapy in young patients with amelogenesis imperfecta. Health Qual Life Outcomes. 2015:13:197-205.

8. Shaw WC. The influence of children's dentofacial appearance on their social attractiveness as judged by peers and lay adults. Am J Orthod. 1981;79:399-415.

9. Kiyak HA. Does orthodontic treatment affect patients' quality of life? J Dent Educ. 2008;72(8):886-94.

10. Dion KK, Berscheid E, Walster E. What is beautiful is good. J Pers Soc Psychol. 1972;24:285-90.

11. Eagly $A H$, Makhijani MG, Ashmore RD, Longo LC. What is beautiful is good, but: a meta-analytic review of research on the physical attractiveness stereotype. Psychol Bull. 1991;110:109-28.

12. Calderini A, Sciara S, Semeria C, Pantaleo G, Polizzi E. Comparative clinical and psychosocial benefits of tooth bleaching: different light-activation of a 38\% peroxide gel in a preliminary case-control study. Clin Case Rep. 2016; 4(8):728-35. https://doi.org/10.1002/ccr3.605.

13. Tecco S, Grusovin MG, Sciara S, Bova F, Pantaleo G, Capparé P. The association between three attitude-related indexes of oral hygiene and secondary implant failures: a retrospective longitudinal study. Int J Dent Hyg. 2017, in press, advance online publication. https://doi.org/10.1111/idh.12300

14. Castelli R, Sciara S, Lambertenghi Deliliers G, Pantaleo G. Biosimilar epoetin alfa increases haemoglobin levels and brings cognitive and socio-relational benefits to elderly transfusion-dependent multiple myeloma patients: results from a pilot study. Ann Hematol. 2017:96(5):779-86. https://doi.org/10. 1007/s00277-017-2950-9

15. Cerulli Mariani G, Favaretti F, Fidanza F, Gatto R. Oral hygiene habits and attitudes in a school population in L'Aquila (Italy). Eur J Paediatr Dent. 2006;7(3):135-41.

16. Sunday O, Akintoye SO, GreenBerg MS. Recurrent aphtous stomatitis. Dent Clin N Am. 2014;58(2):281-97.

17. Cunha-Cruz J, Scott JA, Rothen M, Mancl L, Lawhorn T, Brossel K, Berg J. Salivary characteristics and dental caries: evidence from general dental practices. J Am Dent Assoc. 2013;144(5):e31-40.

18. Sgolastra F, Fidanza F, Carosi D, Petrucci A, Calò G, Gatto R. An interdisciplinary approach to a survey on dental caries in a group of 3-yearolds in Ascoli Piceno (ltaly). Eur J Paediatr Dent. 2010:11(3):137-40.

19. Campus G, Condò SG, Di Renzo G, Ferro R, Gatto R, Giuca MR, Giuliana G, Majorana A, Marzo G, Ottolenghi L, Petti S, Piana G, Pizzi S, Polimeni A, Pozzi A, Sapelli PL, Ugazio A. Italian Society of Paediatric Dentistry. National Italian Guidelines for caries prevention in 0 to 12 years-old children. Eur J Paediatr Dent. 2007;8(3):153-9.

20. Giannoni M, D'Amario M, Gatto R, Barone A. Some tools for the identification of high caries risk individuals. A review. Minerva Stomatol. 2005;54(3):111-27.

21. Tarakji B, Baroudi K, Kharma Y. The effect of dietary habits on the development of the recurrent aphtous stomatitis. Niger Med J. 2012;53(1):9-11.

22. Prati C, Chersoni S, Lucchese A, Pashley DH, Mongiorgi R. Dentin permeability after toothbrushing with different toothpastes. Am J Dent. 1999:12(4):190-3.

23. Pantaleo G. Guidelines for planning and conducting scientific research [Criteri per l'elaborazione di un lavoro scientifico]. Italian Oral Surgery. 2012. (2):47-58. https://doi.org/10.1016/j.ios.2011.12.001. 
24. Pica G, Sciara S, Livi S, Pantaleo G. Ethnic prejudice in person memory: lessened retrieval-induced forgetting of negative traits ascribed to an African-American target. Psicologia Sociale. 2017;12(3):351-62. https://doi. org/10.1482/87890.

25. Giannetti L, Murri A, Vecci F, Gatto R. Dental avulsion: therapeutic protocols and oral health-related quality of life. Eur J Paediatr Dent. 2007:8(2):69-75.

26. Castelli R, Deliliers GL, Colombo R, Gallipoli P, Pantaleo G. Biosimilar epoetin in elderly patients with low-risk myelodysplastic syndromes improves anemia, quality of life, and brain function. Ann Hematol. 2014;93(9):1523-9.

27. Lamperti E, Pantaleo G, Finocchiaro CY, Silvani A, Botturi A, Gaviani P, Sarno L, Salmaggi A. Recurrent brain tumor: the impact of illness on patient's life. Support Care Cancer. 2012;20:1327-32.

28. Pantaleo G, Wicklund RA. Multiple perspectives: social performance beyond the single criterion. Z Sozialpsychol. 2000;31(4):231-42.

29. Brehm JW, Self EA. The intensity of motivation. Annu Rev Psychol. 1989:40: 109-31.

30. Richter M, Gendolla GHE, Wright, RA. Three decades of research on motivational intensity theory: what we have learned about effort and what we still don't know. In A. J. Elliot (Ed.), Advances in motivation science (pp. 149-186). Cambridge: Academic Press. https://doi.org/10.1016/bs.adms. 2016.02.001.

31. Wright RA, Pantaleo G. Effort processes in achieving performance outcomes: interrelations among and roles of core constructs. Behav Brain Sci. 2014; 36(6):705-6.

32. Sciara S, \& Pantaleo, G. Relationships at risk: How the perceived risk of ending a romantic relationship influences the intensity of romantic affect and relationship commitment. Motiv. Emot. 2018;42(1):137-48. https://doi. org/10.1007/s11031-017-9650-6.

33. Pantaleo G, Miron AM, Ferguson MA, Frankowski SD. Effects of deterrence on intensity of group identification and efforts to protect group identity. Motiv Emot Spec Issue Effort. 2014;38(6):855-65.

\section{Submit your next manuscript to BioMed Central and we will help you at every step:}

- We accept pre-submission inquiries

- Our selector tool helps you to find the most relevant journal

- We provide round the clock customer support

- Convenient online submission

- Thorough peer review

- Inclusion in PubMed and all major indexing services

- Maximum visibility for your research

Submit your manuscript at www.biomedcentral.com/submit

) Biomed Central 